\title{
Ground-state and dynamical properties of hard-core bosons on one-dimensional incommensurate optical lattices with harmonic trap
}

\author{
Xiaoming Cai, ${ }^{1}$ Shu Chen, ${ }^{1}$ and Yupeng Wang ${ }^{1}$ \\ ${ }^{1}$ Beijing National Laboratory for Condensed Matter Physics, \\ Institute of Physics, Chinese Academy of Sciences, Beijing 100190, China
}

(Dated: November 2, 2018)

\begin{abstract}
We study properties of the strongly repulsive Bose gas on one-dimensional incommensurate optical lattices with a harmonic trap, which can be deal with by using the exact numerical method through the Bose-Fermi mapping. We first exploit the phase transition of the hard-core bosons in the optical lattices from superfluid-to-Bose-glass phase as the strength of the incommensurate potential increases. Then we study the dynamical properties of the system after suddenly switching off the harmonic trap. We calculate the one-particle density matrices, momentum distributions, the natural orbitals and their occupations for both the static and dynamic systems. Our results indicate that the Bose-glass phase and superfluid phase display quite different properties and expansion dynamics.
\end{abstract}

PACS numbers: 05.30.Rt, 05.30.Jp, 72.15.Rn

\section{INTRODUCTION}

Recently, the technical advances in cold atom trapping allowed the experimental realization of the Anderson localization [1] in quantum matter waves [2, 3]. The good tunability and controllability of optical lattices offer myriad opportunities for studying the disorder effects in ultracold atom system [4]. So far, different techniques have been devised for the introduction of disorder in the ultracold atom system, such as speckle filed patterns in the optical lattice [2, 5], random localized impurities by loading a mixture of two kinds of atoms with heavy and light masses [6], and incommensurate bichromatic optical lattices by superimposing two one-dimensional (1D) optical lattices with incommensurate frequency [3, 7]. In particular, the experiment [7] has provided evidences of the existence of a Bose-glass (BG) phase [8].

Interesting phenomenons are expected to appear in disordered systems when the interplay of disorder and interactions is taken into account. Interactions between atoms can be controllably tuned by Feshbach resonances in ultra-cold atom systems. While disorder can lead to localization of the wave function of a particle, delocalization can arise as the consequence of interactions in some many-body systems. Theoretically, for a repulsive Bose gas it has been predicted that there is a quantum phase transition from a superfluid phase to an insulating BG phase with localized single-particle states as disorder is increased [9 15], however unambiguous observation of the superfluid-Bose-glass transition is still under debate [11, 16]. A lot of attention [17 21] has been payed to investigating the combined role of disorder and interactions in the strongly interacting ultra-cold atomic system. Except numerical or approximate approaches, the exact solution for the many-body systems with the interplay of disorder and interaction are rarely known. In this paper, we study the interacting bosons on the incommensurate optical lattices with harmonic trap in the limit case with infinitely repulsive interaction which can be solved exactly. The 1D Bose gas with infinitely repulsive interaction is known as the hard-core boson (HCB) or Tonks-Girardeau (TG) gas [22], which can be exactly solved via the Bose-Fermi mapping 22$]$ and has attracted intensive theoretical attention [23 25]. Experimental access to the required parameter regime has made the TG gas a physical reality [26, 27]. For the HCB in 1D optical lattices, it is convenient to use the exact numerical approach proposed by Rigol and Muramatsu 28]. Following the exact numerical approach, we calculate the static properties of the hard-core bosons, such as one-particle density matrices, density profiles, momentum distributions, natural orbitals and their occupations, to exploit the superfluid-to-BG phase transition for the systems in incommensurate optical lattices with harmonic confining trap. Furthermore, we study the nonequilibrium dynamical properties of expanding clouds of hard-core bosons on the 1D incommensurate lattices after turning off the harmonic trap suddenly. We find that the expansion dynamics for the superfluid phase and BG phase exhibit quite different behaviors, which may serve as a signature for experimentally detecting the transition from superfluidto-BG phase.

The paper is organized as follows. In Section II, we present the model and the exact approach used in this paper. In Section III, we show properties of the groundstate for hard-core bosons on the incommensurate optical lattice with a harmonic trapping potential. Section IV is devoted to studying the nonequilibrium dynamics of the system after the harmonic trap is suddenly switched off. Finally, a summary is presented in Section V.

\section{MODEL AND METHOD}

In the present section we describe the exact approach which we used to study $1 \mathrm{D}$ hard-core bosons on the incommensurate lattice with an additional harmonic trap. Under the single-band tight-binding approximation, the 
system of $N$ hard-core bosons in the 1D optical lattice can be described by the following Hamiltonian:

$$
H=-t \sum_{i}\left(b_{i}^{\dagger} b_{i+1}+\text { H.c. }\right)+\sum_{i} V_{i} n_{i}^{b},
$$

where $b_{i}^{\dagger}\left(b_{i}\right)$ is the creation (annihilation) operator of the boson which fulfills the hard-core constraints [28], i.e., the on-site anticommutation $\left(\left\{b_{i}, b_{i}^{\dagger}\right\}=1\right)$ and $\left[b_{i}, b_{j}^{\dagger}\right]=0$ for $i \neq j ; n_{i}^{b}$ is the bosonic particle number operator; $t$ is the hopping amplitude set to be the unit of the energy $(t=1) ; V_{i}$ is given by

$$
V_{i}=V_{I} \cos (\alpha 2 \pi i+\delta)+V_{H}\left(i-i_{0}\right)^{2} .
$$

Here $V_{I}$ is the strength of incommensurate potential with $\alpha$ as an irrational number characterizing the degree of the incommensurability and $\delta$ an arbitrary phase (in our calculation it is chosen to be zero for convenience, without loss of generality), $V_{H}$ is the strength of harmonic trap and $i_{0}$ is the position of the vale of the harmonic trap.

In order to calculate the properties of hard-core bosons, it is convenient to use the Jordan-Wigner transformation [29] (JWT) or Bose-Fermi mapping for the lattice model

$$
b_{j}^{\dagger}=f_{j}^{\dagger} \prod_{\beta=1}^{j-1} e^{-i \pi f_{\beta}^{\dagger} f_{\beta}}, b_{j}=\prod_{\beta=1}^{j-1} e^{+i \pi f_{\beta}^{\dagger} f_{\beta}} f_{j},
$$

which maps the Hamiltonian of hard-core bosons into the Hamiltonian of noninteracting spinless fermions

$$
H_{F}=-\sum_{i}\left(f_{i}^{\dagger} f_{i+1}+\text { H.c. }\right)+\sum_{i} V_{i} n_{i}^{f},
$$

where $f_{i}^{\dagger}\left(f_{i}\right)$ is the creation (annihilation) operator of the spinless fermion and $n_{i}^{f}$ is the particle number operator. The ground-state wave function of the system with $N$ spinless free fermions can be obtained by diagonalizing Eq.(44) and can be represented as

$$
\left|\Psi_{F}^{G}\right\rangle=\prod_{n=1}^{N} \sum_{i=1}^{L} P_{i n} f_{i}^{\dagger}|0\rangle,
$$

where $L$ is the number of the lattice sites, $N$ is the number of fermions (same as bosons), and the coefficients $P_{i n}$ are the amplitude of the $n$-th single-particle eigenfunction at the $i$-th site which can form an $L \times N$ matrix $P$ [28].

In order to get the static properties of the groundstate, we calculate the one-particle Green function for the hard-core bosons defined by

$$
G_{i j}=\left\langle\Psi_{H C B}^{G}\left|b_{i} b_{j}^{\dagger}\right| \Psi_{H C B}^{G}\right\rangle=\left\langle\Psi^{A} \mid \Psi^{B}\right\rangle,
$$

where $\left|\Psi_{H C B}^{G}\right\rangle$ is the ground-state of hard-core bosons, and $\left\langle\Psi^{A}\right|=\left(f_{i}^{\dagger} \prod_{\beta=1}^{i-1} e^{-i \pi f_{\beta}^{\dagger} f_{\beta}}\left|\Psi_{F}^{G}\right\rangle\right)^{\dagger}, \quad\left|\Psi^{B}\right\rangle=$ $f_{j}^{\dagger} \prod_{\gamma=1}^{j-1} e^{-i \pi f_{\gamma}^{\dagger} f_{\gamma}}\left|\Psi_{F}^{G}\right\rangle$. Explicitly the state $\left|\Psi^{A}\right\rangle$ can be represented as $\left|\Psi^{A}\right\rangle=\prod_{n=1}^{N+1} \sum_{l=1}^{L} P_{l n}^{A} f_{l}^{\dagger}|0\rangle$ with $P_{l n}^{A}=-P_{l n}$ for $l \leq i-1, P_{l n}^{A}=P_{l n}$ for $l \geq i$ with $n \leq N, P_{i N+1}^{A}=1$ and $P_{l N+1}^{A}=0(l \neq i)$. Similarly we can get $P^{B}$ for the state $\left|\Psi^{B}\right\rangle$ with the replacement of $i$ by $j$. The Green function is a determinant dependent on the $L \times(N+1)$ matrices $P^{A}$ and $P^{B}[28]$

$$
G_{i j}=\left\langle\Psi^{A} \mid \Psi^{B}\right\rangle=\operatorname{det}\left[\left(P^{A}\right)^{\dagger} P^{B}\right] .
$$

The one-particle density matrix can be evaluated from the relation

$$
\rho_{i j}=\left\langle b_{i}^{\dagger} b_{j}\right\rangle=G_{i j}+\delta_{i j}\left(1-2 G_{i i}\right) .
$$

Alternatively one can use the method proposed by Pareder et al. 26] to calculate the one-particle density matrix directly. The momentum distribution is defined by the Fourier transform with respect to $i-j$ of the oneparticle density matrix with the form

$$
n(k)=\frac{1}{L} \sum_{i, j=1}^{L} e^{-i k(i-j)} \rho_{i j},
$$

where $k$ denotes the momentum. The eigenfunctions or natural orbitals $\left(\phi_{i}^{\eta}\right)$ of the one-particle density matrix [30] can be obtained by solving

$$
\sum_{j=1}^{L} \rho_{i j} \phi_{j}^{\eta}=\lambda_{\eta} \phi_{i}^{\eta},
$$

which can be understood as being effective single particle states with occupations $\lambda_{\eta}$. For noninteracting bosons, all the particles occupy in the lowest natural orbital and bosons are in the BEC phase at zero temperature, however only the quasi condensation exists for the 1D hardcore bosons. For hard-core bosons in the incommensurate optical lattice, the strong incommensurate potential can destroy the quasi-BEC.

The nonequilibrium dynamical properties of expanding clouds of hard-core bosons on 1D incommensurate lattice after turning off the harmonic trap suddenly can be also calculated through the equal time Green function. With the JWT, we can express the equal time Green function for the expanding hard-core bosons as

$$
\begin{aligned}
G_{i j}(t) & =\left\langle\Psi_{H C B}^{G}(t)\left|b_{i} b_{j}^{\dagger}\right| \Psi_{H C B}^{G}(t)\right\rangle \\
& =\left\langle\Psi_{F}^{G}(t)\left|\prod_{\beta=1}^{i-1} e^{i \pi f_{\beta}^{\dagger} f_{\beta}} f_{i} f_{j}^{\dagger} \prod_{\gamma=1}^{j-1} e^{-i \pi f_{\gamma}^{\dagger} f_{\gamma}}\right| \Psi_{F}^{G}(t)\right\rangle,
\end{aligned}
$$

where $\left|\Psi_{H C B}^{G}(t)\right\rangle$ is the wave function of the hard-core bosons at $t$ after turning off the harmonic trap and $\left|\Psi_{F}^{G}(t)\right\rangle$ is the corresponding one for the noninteracting fermions. The wave function $\left|\Psi_{F}^{G}(t)\right\rangle$ can be easily calculated with the initial wave function $\left|\Psi_{F}^{G}\right\rangle$

$$
\left|\Psi_{F}^{G}(t)\right\rangle=e^{-i H_{F}^{\prime} t}\left|\Psi_{F}^{G}\right\rangle=\prod_{n=1}^{N} \sum_{l=1}^{L} P_{l n}(t) f_{l}^{\dagger}|0\rangle,
$$



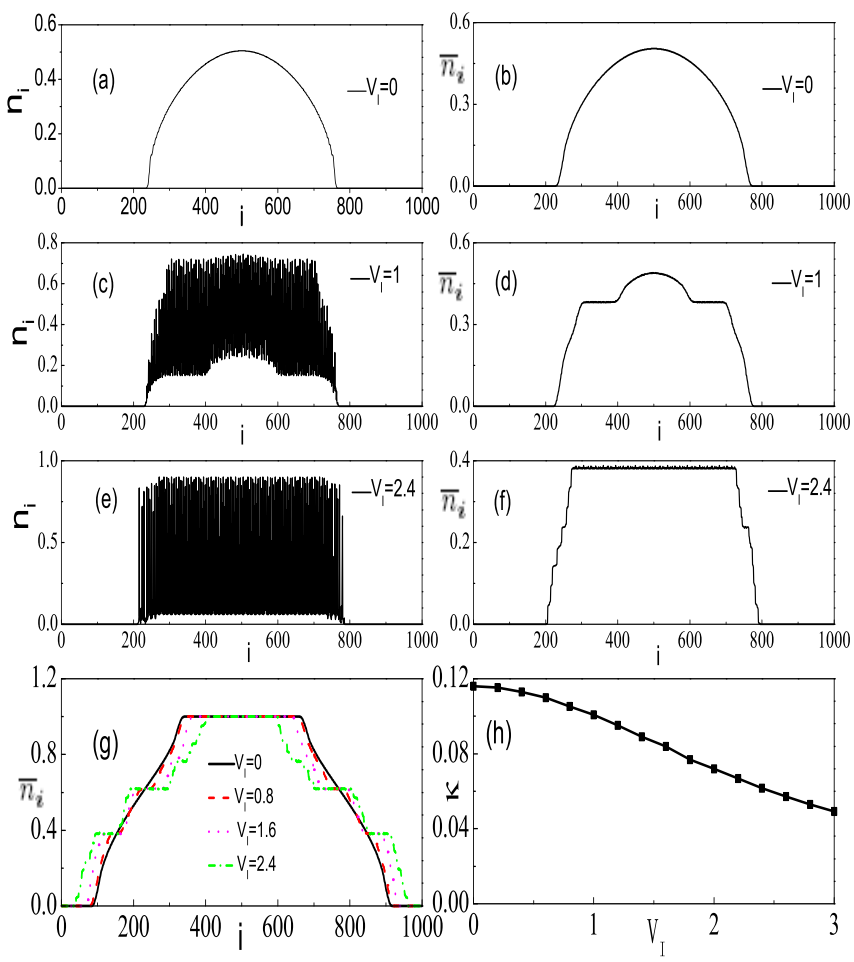

FIG. 1: (Color online) The density profiles(a,c,e) and the local average density distributions $(b, d, f)$ for the systems with 1000 sites; 200 bosons; $V_{H}=3 \times 10^{-5} ; \alpha=(\sqrt{5}-1) / 2$; and $(\mathrm{a}, \mathrm{b}) V_{I}=0,(\mathrm{c}, \mathrm{d}) V_{I}=1$, and $(\mathrm{e}, \mathrm{f}) V_{I}=2.4$. (g): The local average density distributions for the systems with 1000 sites, 600 bosons, $V_{H}=3 \times 10^{-5}, \alpha=(\sqrt{5}-1) / 2$ and different $V_{I}$. (h): The mean particle fluctuation vs $V_{I}$ for the systems with 1000 sites, 200 bosons, $V_{H}=3 \times 10^{-5}, \alpha=(\sqrt{5}-1) / 2$.

which is still a product of time-dependent single-particle states, where $\hbar$ has been set to be unit of time in the evolution operator, $H_{F}^{\prime}$ is the $H_{F}$ in Eq. (44) with $V_{H}=0$, and $P(t)$ is the matrix of $\left|\Psi_{F}^{G}(t)\right\rangle$ in the same way as $\left|\Psi_{F}^{G}\right\rangle$. Then we can use the method described above to calculate the equal time Green function, yet we can get the one-particle density matrix, density profile, momentum distribution, natural orbitals and their occupations.

\section{STATIC PROPERTIES OF HARD-CORE BOSONS CONFINED IN HARMONIC TRAP}

Hard-core bosons in harmonic trap without the incommensurate potential have been studied by Rigol and $\mathrm{Mu}-$ ramatsu [28] in detail. Quantitatively they characterize the system in a harmonic trap with the length scale set by combination lattice-confining potential $\zeta=\left(V_{H} / t\right)^{1 / 2}$, and the associated characteristic density $\widetilde{\rho}=N / \zeta[31]$. They found that there is a critical characteristic density $\left(\widetilde{\rho}_{c} \sim 2.6-2.7\right)$ in the system, for $\widetilde{\rho}<\widetilde{\rho}_{c}$ the whole system is in the superfluid phase at zero temperature, and for $\tilde{\rho}>\widetilde{\rho}_{c}$ there is a phase separation in the system with a Mott insulating plateau in the middle of the trap with filling factor equaling one surrounding by the superfluid phase on the two sides. In this paper we mainly study the influence of the incommensurate potential which acts as the quasi-random potential and leads to localization of particles. We focus our study on systems with low characteristic density $\left(\widetilde{\rho}<\widetilde{\rho}_{c}\right)$. For system with high characteristic density the properties are similar to the one with low characteristic density except that there is a Mott insulator plateau in the system. The Mott insulator plateau is not dramatically influenced by the incommensurate potential because the particles in the Mott phase are already localized with one-particle density matrix $\rho_{i j}=\delta_{i j}$. Without especially illustration, properties studied in this section are for systems with low characteristic density.

Following the method described in the above section, we can get the one-particle density matrix. The density profile is given by the diagonal elements of the oneparticle density matrix, i.e., $n_{i}=\rho_{i i}$. In the low characteristic density region, the density profiles for three different strength of the incommensurate potential are shown in Fig.1. In the absence of the incommensurate potential $\left(V_{I}=0\right)$, the density profile is shown in Fig.1(a) with all the bosons being in the superfluid phase at the vale of the harmonic trap. As $V_{I}$ increases but is still small, the density profiles basically have the arc shape, but there are a lot of drastic oscillations in the profiles induced by the incommensurate potential. The amplitude of the oscillations becomes more and more large with the increase in $V_{I}$. When $V_{I}$ becomes large enough, the density almost oscillates in the range of 0 to 1 . The density profile looks like a belt at the center of harmonic trap. Additionally, the width of the density profile almost does not change with the change of $V_{I}$. For system in high characteristic density region, the Mott plateau in the density profile basically does not change with the increase in $V_{I}$ because the particles in this phase are already localized. However the superfluid regions exhibit similar behavior as the system in low characteristic density region.

Since there are many drastic oscillations in the density distributions, it is hard to tell that in which area the density is high in a density profile. In order to reduce the drastic oscillations, we define the local average density $\bar{n}_{i}$ as

$$
\bar{n}_{i}=\sum_{j=-M}^{M} n_{i+j} /(2 M+1),
$$

where $2 M+1$ is the length to count the local average density with $M \ll L$. In this work, we set $M=10$. The local average density distributions for three different values of $V_{I}$ are also shown in Fig 1. When $V_{I}=0$, the local average density distribution is almost the same as the density profile with no drastic oscillations. As $V_{I}$ increases, plateaus appear at the shoulders of the arc (see Fig 1(d)), then become wider and wider. Such kind of plateau is induced by the disorder, and thus we call it 
Anderson plateau hereafter. When $V_{I}>2$, the shape of the local average density does not change with the increase in $V_{I}$. In Fig 1 (e) and (f), the density distribution and the local average density distribution for $V_{I}=2.4$ are displayed. The number of the plateaus and their locations are related to the particle number and the strength of the harmonic trap. In Fig 1 there happened to be only two plateaus. For system with high characteristic density, the local average density distributions for different $V_{I}$ are shown in Fig प(g). The Mott insulator plateau always exists in the trap center as $V_{I}$ increases, except that the edges of the plateau are more and more dissolved into superfluid phase when $V_{I}<2$. Meanwhile new plateaus appear at the two sides of the distribution, which is similar to the one with low characteristic density as $V_{I}$ increases. The Mott plateau in the trap center is characterized by $n_{i}=1$ which does not oscillate against the particle density in the Anderson plateaus due to the existence of the incommensurate potential.

The mean particle fluctuation for various value of $V_{I}$ is shown in Fig 1 (h). It is defined by $\kappa=\sum_{i=1}^{L} \kappa_{i} / L$ with $\kappa_{i}$ being the local particle fluctuation $\kappa_{i} \equiv\left\langle\hat{n}_{i}^{2}\right\rangle-n_{i}^{2}=$ $n_{i}-n_{i}^{2}$ for hard-core bosons. We can see that $\kappa$ decreases as $V_{I}$ increases because of the incommensurate lattice making the bosons difficult to hop. For system in high characteristic density region, the local particle fluctuation for particles in the Mott insulator plateau always equals zero as $n_{i}=1$ for any $V_{I}$, whereas the particle fluctuation is not equal to zero for particles in Anderson plateaus. So we can distinguish the Mott region from the superfluid region or Bose-glass region by the local particle fluctuation.

In Fig 2(a,b), we show the one-particle density matrices for different value of $V_{I}$ and site. In the absence of the incommensurate potential $\left(V_{I}=0\right)$, the density matrix has a power-law decay with exponent $-1 / 2[28]$. As $V_{I}$ increases but is still small, the density matrices still have the power-law decay, but the exponents are smaller than $-1 / 2$ and have lots of oscillations induced by the incommensurate potential. Power-law decay of the density matrix is the character of the system in superfluid phase, so when $V_{I}$ is small, the system in the superfluid phase. But things change when the Anderson plateaus appear in the local average density distribution as $V_{I}$ increases. The one-particle density matrices for the sites in Anderson plateaus have an exponential-law decay, while the ones for the sites out of the Anderson plateaus still have the power-law decay. Different from the case without the harmonic confining trap [15], the systems are not in a uniform phase. When $V_{I}>2$, the local average density distribution only consists of several Anderson plateaus, and the density matrix has the exponential-law decay which is the character of the system in Bose-glass phase. For system with $\widetilde{\rho}>\widetilde{\rho}_{c}$, the one-particle density matrix is given by $\rho_{i j}=\delta_{i j}$ for $i, j$ in the Mott insulator plateau, and the one for $i, j$ on the shoulders in the density profile behaves like the density matrix of the system in low characteristic region. In Fig 2 (c), we show the
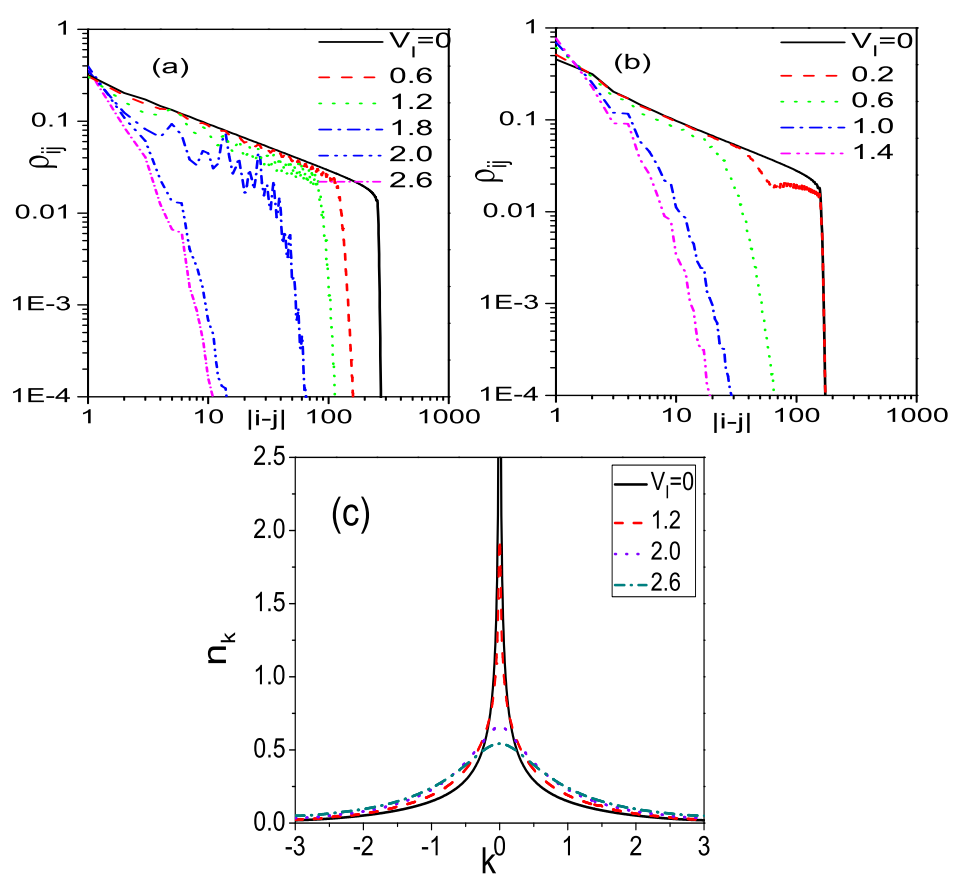

FIG. 2: (Color online) One-particle density matrices for systems with 1000 sites; 200 bosons; $V_{H}=3 \times 10^{-5} ; \alpha=$ $(\sqrt{5}-1) / 2$; and $(\mathrm{a}) i=501,(\mathrm{~b}) i=601$. (c): The momentum distributions for systems with 1000 sites, 200 bosons, $V_{H}=3 \times 10^{-5}, \alpha=(\sqrt{5}-1) / 2$.

momentum distributions for different value of $V_{I}$. When $V_{I}$ is small, the momentum distribution is similar to the case of $V_{I}=0$ and has a sharp peak at $k=0$ reflecting the coherence of the system. As $V_{I}$ increases further, the peak becomes more and more shallow and the distribution spreads wider. When $V_{I}>2$, there is almost no obvious peak. The system is in the Bose-glass phase with all the effective single-particle states being localized states, and the coherence of the system becomes small.

Now we study the properties of the natural orbitals and their occupations for the systems with the low characteristic density. The occupations of the natural orbitals for systems with different $V_{I}$ are shown in Fig 3 , The occupations are plotted versus the orbital numbers $\eta$, and ordered starting from the highest occupied one. When $V_{I}$ is small, the occupation distribution has a sharp single peak at $\eta=1$ which is the feature of bosons in contrast to the step function for fermions. With the increase in $V_{I}$, the occupation of the lowest natural orbital $\left(\lambda_{1}\right)$ decreases. When the system is in the Bose-glass phase for $V_{I}>2$, no an obvious peak appears in the lowest natural orbital. Additionally we can find that there is a discontinuation in the occupation distribution at $\eta=N$ when $V_{I}>2$. We use $Z=\lambda_{N}-\lambda_{N+1}$ [15] to characterize the discontinuation for a Bose gas with $N$ particles. In Fig $3($ d) $Z$ versus $V_{I}$ is plotted. There is an obvious change around $V_{I}=2$. The difference of the occupations between systems with the low and high characteristic density is that 

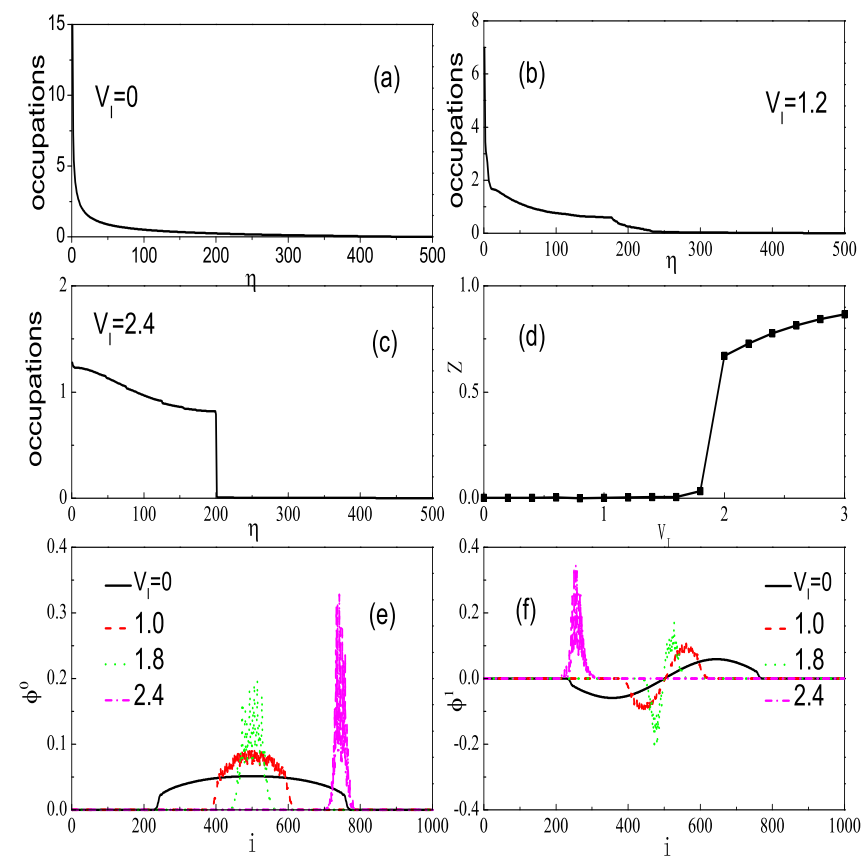

FIG. 3: Occupations of the natural orbitals for systems with 1000 lattice sites; 200 bosons; $\alpha=(\sqrt{5}-1) / 2 ; V_{H}=3 \times$ $10^{-5}$; and (a) $V=0,(\mathrm{~b}) V=1.2$, and (c) $V=2.4$. (d): The amplitude of the discontinuation $(Z)$ which we define as $Z=$ $\lambda_{N}-\lambda_{N+1}$ relates to $V_{I}$ for systems with 1000 lattice sites, 200 bosons $\alpha=(\sqrt{5}-1) / 2$ and $V_{H}=3 \times 10^{-5}$. (e,f): Profiles of the two lowest natural orbitals for systems with 1000 lattice sites, 200 bosons, $\alpha=(\sqrt{5}-1) / 2$ and $V_{H}=3 \times 10^{-5}$.

there is a plateau in the occupation for system in high $\widetilde{\rho}$ region due to the existence of a Mott insulator plateau. Such a plateau is basically not changed as $V_{I}$ increases. In Fig 3 we also show the effect of the incommensurate potential on the natural orbitals. We plot profiles of two lowest natural orbitals for different value of $V_{I}$. Without the incommensurate lattice, the natural orbitals are similar to the ones of hard-core bosons in the harmonic trap without lattice. As $V_{I}$ increases but is still small, the natural orbitals have similar shapes as the case of $V_{I}=0$ ones, but the widths of the wave functions become smaller and smaller due to the particles becoming more localized. When $V_{I}>2$, all the natural orbitals spread only over a few lattice sites, and they become typical Anderson localized state [15, 32]. The system is in the Bose-glass phase with exponential-law decay oneparticle density matrix when $V_{I}>2$. For system with high $\widetilde{\rho}$, the amplitude of the natural orbitals in the Mott insulator region has to be zero (see Ref. 28]).

\section{DYNAMICAL PROPERTIES OF HARD-CORE BOSONS}

In this section, we study the nonequilibrium dynamical properties of expanding clouds of hard-core bosons on

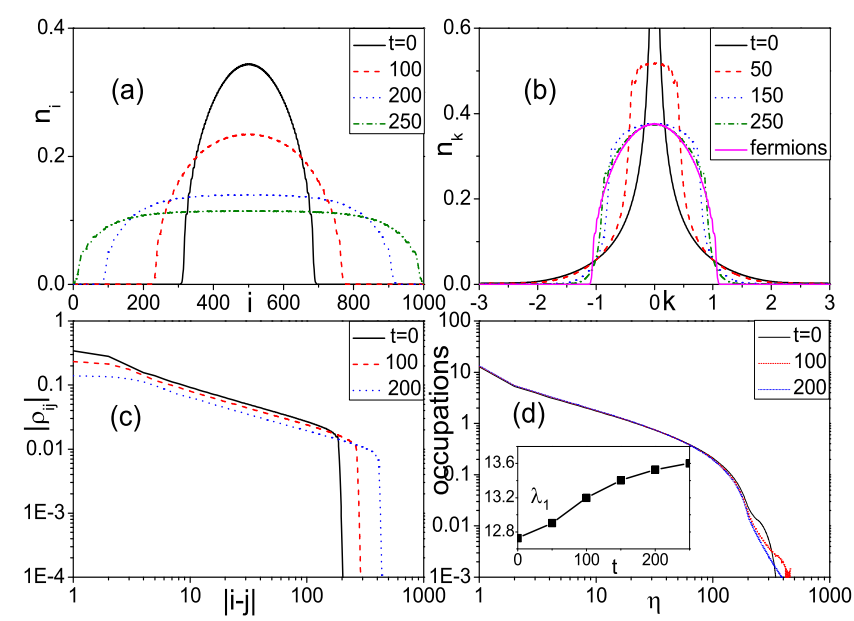

FIG. 4: (Color online) The evolution of the density profile(a), momentum distribution(b), one-particle density ma$\operatorname{trix}(\mathrm{c})$ with $i=501$, occupations(d) of the natural orbitals for system with 1000 lattice sites, 100 bosons, $\alpha=(\sqrt{5}-1) / 2$, $V_{H}=3 \times 10^{-5}$, and $V_{I}=0 . \quad t$ is the time after turning off the trap. The "fermions" curve is the momentum distribution for system with 1000 lattice sites, 100 free fermions, $\alpha=(\sqrt{5}-1) / 2, V_{H}=3 \times 10^{-5}$, and $V_{I}=0$. Insert of $(\mathrm{d})$ : The occupation of the lowest natural orbital vs $t$ for system with 1000 lattice sites, 100 bosons, $\alpha=(\sqrt{5}-1) / 2, V_{H}=3 \times 10^{-5}$, and $V_{I}=0$.

1D incommensurate lattice after turning off the harmonic trap suddenly. We first consider the situations that the systems are in the superfluid phase with small $V_{I}$ before turning off the trap. The system with $V_{I}=0$ has been studied by Rigol and Muramatsu [31]. In order to see clearly the effect of a nonzero value of $V_{I}$ and provide an example for comparison, we first present results for the case of $V_{I}=0$ following [31]. As shown in Fig 4, the evolution of the density profile is ordinary, i.e., after turning off the harmonic trap the density profile spreads more and more wider as the time increases. As for the evolution of the momentum distribution, shortly after turning off the trap, the peak at $k=0$ disappears. After a long time, the momentum distribution has similar shape as the distribution of the noninteracting fermions which does not change during the expansion [31]. Although the momentum distribution displays the behavior of fermionization [31], the modulus of one-particle density matrix still has the power-law decay which is the character of the bosons in superfluid phase. Here we note that the elements of the density matrix are complex numbers after turning off trap. The occupations of natural orbitals basically remain unchanged, but the occupations of the lowest natural orbitals slightly increase during the expansion. This can be understood as a result due to the increase in the size of the expanding atomic cloud. The evolution of the natural orbitals has similar behavior as the density profile which becomes more and more wider. 

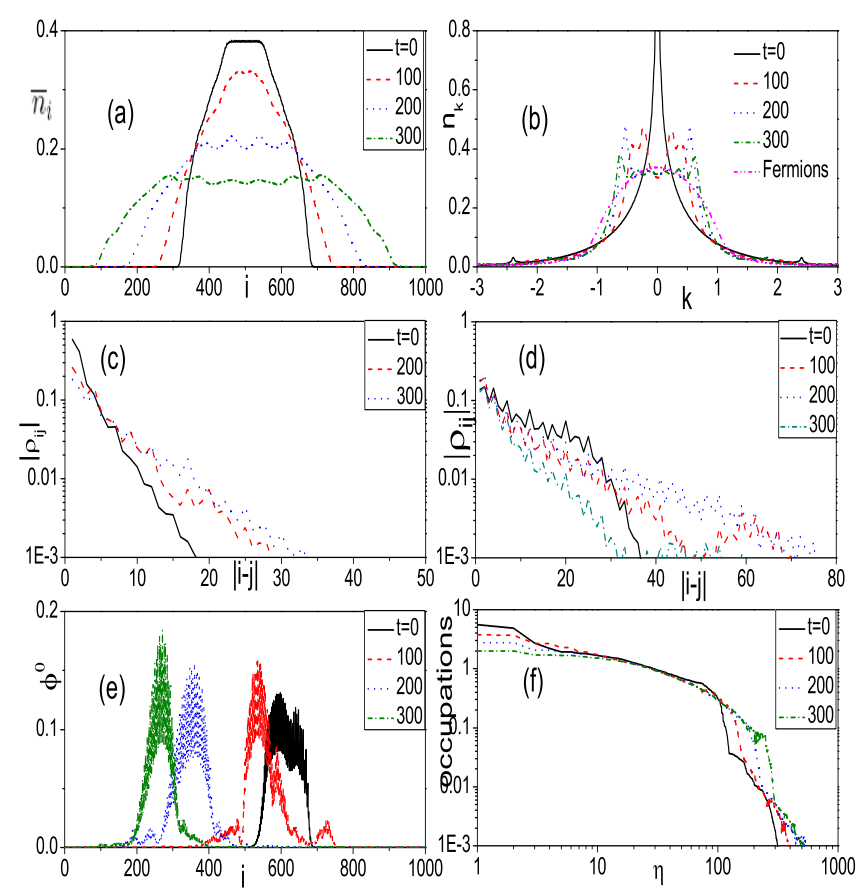

FIG. 5: (Color online) The evolution of the local average density distribution(a), momentum distribution(b), one-particle density matrix with (c) $i=501,(\mathrm{~d}) i=650$, occupations(f) of the natural orbitals for system with 1000 lattice sites, 100 bosons, $\alpha=(\sqrt{5}-1) / 2, V_{H}=3 \times 10^{-5}$, and $V_{I}=1$. (e): The lowest natural orbitals at different time during expansion for systems with 1000 lattice sites, 100 bosons, $\alpha=(\sqrt{5}-1) / 2$, $V_{H}=3 \times 10^{-5}$, and $V_{I}=1$.

Now we consider the evolution of the system in the presence of the incommensurate potential. For the case that the system in a weak incommensurate potential is initially in the superfluid phase, the evolution of the density profile is similar to the one with $V_{I}=0$ after turning off the harmonic trap. To give a concrete example, in Fig 5 we present the evolution of the local average density for the system with $V_{I}=1$. The density profile becomes more and more wider and the plateaus vanish during expansion. Comparing to the density profile in Fig 4, we can see that the expansion is much more slow as the incommensurate potential is added in which acts as a pseudo-random potential and prevents movement of particles. The evolution of the momentum distribution is shown in Fig 5 (b). Shortly after turning off the harmonic trap, the peak at $k=0$ disappears. In the presence of the incommensurate potential, there appear some small peaks on the distribution. As time increases, the peaks of the momentum distribution become small, and the momentum distribution evolves to the one similar to the corresponding noninteracting fermions. The evolution of the one-particle density matrix is shown in Fig 5 (c,d). The density matrix $\left|\rho_{i j}\right|$ has the exponential-
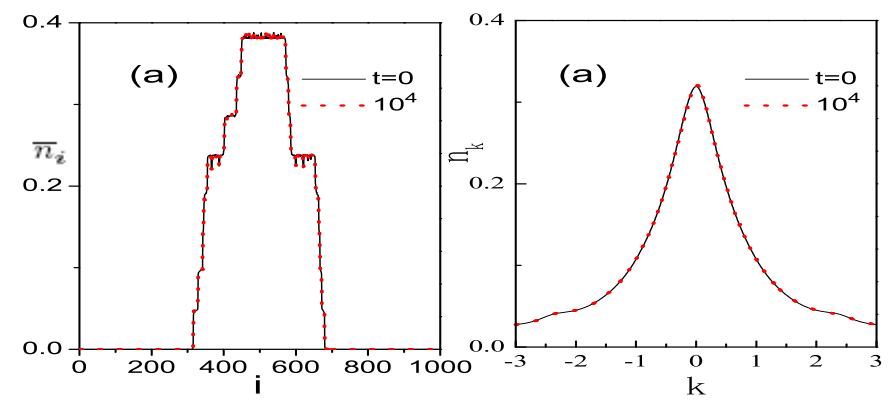

FIG. 6: (Color online) The evolution of the local average density distribution(a) and momentum distribution(b) for system with 1000 lattice sites, 100 bosons, $\alpha=(\sqrt{5}-1) / 2$, $V_{H}=3 \times 10^{-5}$, and $V_{I}=2.5$.

law decay at $t=0$ for $i$ in the Anderson plateaus. For a finite $t$, it still has the exponential-law decay despite the disappearance of Anderson plateau. For $i$ out of the Anderson plateaus, $\left|\rho_{i j}\right|$ has the power-law decay at $t=0$ , and it also has the exponential-law decay after turning off the harmonic trap. In Fig.5(f) we show the lowest natural orbitals for different time during expansion. The natural orbitals for system at different time have different shapes, which can be attributed to the scattering of initial wave function by the incommensurate potential. Correspondingly the occupations of the natural orbitals are different at different time during expansion.

Finally, we study the evolution of system in the BG phase with $V_{I}>2$. When $V_{I}>2$, the system is in the BG phase with all the effective single-particle states being Anderson-localized states. After turning off the harmonic trap, the evolution of the system is very slow, and basically remains unchanged even after a long time. As an example, we show the evolutions of the local average density and momentum distribution in Fig 6, in which we cannot find the difference between the initial one and the one after a long time. This clearly indicates that the system is completely pinned down by the strong incommensurate potential. The asymmetry of the local average density is caused by the asymmetry of the incommensurate lattice.

\section{SUMMARY}

In summary, we have studied the properties of hardcore bosons on incommensurate optical lattices with harmonic confining trap. Using the Bose-Fermi mapping and the exact numerical method proposed by Rigol and $\mathrm{Mu}-$ ramatsu [28, 31], we calculate the one-particle density matrices, momentum distributions, natural orbitals and their occupations for both the static system and the dynamic system at different time. Particularly, we exploit the phase transition from superfluid to the localized BG phase as the strength of the incommensurate potential increases from weak to strong, and the nonequilibrium dynamical properties of expanding clouds of hard-core 
bosons on the incommensurate lattice after turning off the harmonic trap suddenly. For the optical lattice with harmonic confining trap, the density profiles show obvious different characters for systems with weak, intermediate and strong incommensurate potential. When the strength of the incommensurate potential becomes strong enough, Anderson plateaus are founded in the density distribution. The shape of the local average density profile shall not change with the increase in the strength of incommensurate potential if it exceeds a critical value of $V_{I}=2$. When the harmonic trap is suddenly switched off, the expansion dynamics for the systems with $V_{I}<2$ and with $V_{I}>2$ also exhibits quite different behaviors. All of these quantities give clear signature that there exists a superfluid-to-Bose glass phase transition in the sys- tem when the strength of incommensurate potential exceeds $V_{I}=2$. Our study provides an exact static and dynamic example which unambiguously exhibits the transition from superfluid-to-Anderson-insulator in the incommensurate optical lattice.

\section{Acknowledgments}

This work has been supported by NSF of China under Grants No.10821403 and No.10974234, programs of Chinese Academy of Science, 973 grant No.2010CB922904 and National Program for Basic Research of MOST.
[1] P. W. Anderson, Phys. Rev. 109, 1492 (1958).

[2] J. Billy et al., Nature (London) 453, 891 (2008).

[3] G. Roati et al., Nature (London) 453, 895 (2008).

[4] L. Sanchez-Palencia and M. Lewenstein, Nature Physics 6, 87 (2010).

[5] J. E. Lye et al., Phys. Rev. Lett. 95, 070401 (2005); D. Clément et al., Phys. Rev. Lett. 95, 170409 (2005); C. Fort et al., Phys. Rev. Lett. 95, 170410 (2005); T. Schulte et al., Phys. Rev. Lett. 95, 170411 (2005); Y. P. Chen et al., Phys. Rev. A. 77, 033632 (2008).

[6] U. Gavish and Y. Castin, Phys. Rev. Lett. 95, 020401 (2005).

[7] L. Fallani, J. E. Lye, V. Guarrera, C. Fort, and M. Inguscio, Phys. Rev. Lett. 98, 130404 (2007).

[8] M. P. Fisher et al., Phys. Rev. B. 40, 546 (1989).

[9] T. Giamarchi and H. J. Schulz, Phys. Rev. B. 37, 325 (1988); Europhys. Lett. 3, 1287 (1987).

[10] M. E. Fisher, M. N. Barber, and D. Jasnow, Phys. Rev. A. 8, 1111 (1973); W.Krauth, Phys. Rev. B. 44, 9772 (1991).

[11] D. Delande and J. Zakrzewshi, Phys. Rev. Lett. 102, 085301 (2009).

[12] L. Fontanesi, M. Wouters, V. Savona, Phys. Rev. Lett. 103, 030403 (2009).

[13] A. De Martino, M. Thorwart, R. Egger, and R. Graham, Phys. Rev. Lett. 94, 060402 (2005).

[14] K. V. Krutitsky, M. Thorwart, R. Egger, R. Graham, Phys. Rev. A 77, 053609 (2008).

[15] X. Cai, S. Chen, and Y. Wang, Phys. Rev. A 81, 023626
(2010).

[16] B. Damski et al., Phys. Rev. Lett. 91, 080403 (2003).

[17] V. Gurarie, L. Pollet, N. V. Prokofev, B. V. Svistunov, and M. Troyer, arXiv: 0909.4593; L. Pollet, N. V. Prokofev, B. V. Svistunov, and M. Troyer, arXiv: 0903.3867. B. V. Svistunov, Phys. Rev. B 54, 16131 (1996).

[18] G. Roux et al., Phys. Rev. A. 78, 023628 (2008).

[19] X. Deng et al., Phys. Rev. A. 78, 013625 (2008).

[20] T. Roscilde, Phys. Rev. A. 77, 063605 (2008).

[21] G. Orso, Phys. Rev. Lett. 99, 250402 (2007).

[22] M. Girardeau, J. Math. Phys 1, 1268 (1960).

[23] M. Girardeau et al., Phys. Rev. A. 63, 033601 (2001).

[24] A. Minguzzi et al., Phys. Lett. A. 294, 222 (2002).

[25] D. M. Gangardt, J. Phys. A. 27, 9335 (2004).

[26] B. Paredes et al., Nature(London) 429, 277 (2004).

[27] T. Kinoshita et al., Science 305, 1125 (2004).

[28] M. Rigol and A. Muramatsu, Phys. Rev. A. 72, 013604 (2005); M. Rigol and A. Muramatsu, Phys. Rev. A. 70, 031603(R) (2004).

[29] P. Jordan and E. Wigner, Z.Phys. 47, 631 (1928).

[30] O. Pensose and L. Onsager, Phys. Rev. 104, 576 (1956).

[31] M. Rigol and A. Muramatsu, Phys. Rev. Lett 93, 230404 (2004), M. Rigol and A. Muramatsu, Phys. Rev. Lett 94, 240403 (2005).

[32] S. Das Sarma, S. He, and X. C. Xie, Phys. Rev. Lett. 61, 2144 (1988); S. Das Sarma, S. He, and X. C. Xie, Phys. Rev. B. 41, 5544 (1990). 